SESSION 7

ENERGY CONSIDERATIONS AND THE

ELECTRODYNAMIC LINK

BETWEEN THE PULSAR AND THE NEBULA 


\title{
7.1 RELATIONSHIP BETWEEN PULSAR AND NEBULA
}

\author{
L. WOLTJER \\ Department of Astronomy, Columbia University, \\ New York, N.Y., U.S.A.
}

\begin{abstract}
The magnetic field and the relativistic electrons in the Crab Nebula cannot have originated at the time of the supernova explosion. The energy density in the magnetic field is so large that it must have been generated using the energy supply in the pulsar. The energies of the electrons are so high, and their lifetimes correspondingly are so short, that they must have been accelerated, again using the pulsar energy. The efficiency of these processes must be high, but there is an adequate energy supply.
\end{abstract}

\section{Introduction}

For a long time the origin of the nebular magnetic field and the acceleration process for relativistic electrons have posed the most difficult problems in attempts towards an understanding of the Crab Nebula. Though much remains to be done, it seems that the discovery of the pulsar in the Nebula has brought us close to a solution of these problems.

\section{The Nebular Magnetic Field}

Several modes of origin can be excluded. The magnetic field cannot have resulted from a simple expansion of the field in the presupernova; during a spherical expansion the ratio of gravitational to magnetic energy remains constant and this ratio now being $10^{-6}$ the field never could have been contained in a stable object. A similar problem is encountered if one considers an inverse square field drawn out from (but still anchored in) the central star. Turbulent amplification of a weak field is in principle possible, but appears incompatible with the filamentary kinematics; the expansion velocities far exceed those of non radial motions.

Recently Goldreich and Julian (1969) have concluded that the electric fields associated with the rotation of a neutron star are sufficiently strong to lift electrons and positive ions from its surface. Because the positive and negative particles follow different trajectories, large scale poloidal currents may arise (if there is no background medium with high conductivity) which would result in mainly toroidal magnetic fields. Crude estimates suggest that fields of the order of $10^{-4} \mathrm{G}$ may be obtained.

An alternative suggestion has been made by Gunn and Ostriker (1969) namely that no static magnetic field is present and that the relativistic electrons radiate in the fields of electromagnetic waves emitted by the rotating magnetic neutron star. This latter model is in a sense a limiting case, strictly valid if the pulsar is surrounded by a vacuum. Only a fully self consistent solution - which will not be easily obtained - can tell whether the real situation is closer to the wave solution or to 
the static case, that is whether displacement currents or charged particle currents dominate.

\section{Particle Acceleration}

The relativistic electrons in the Crab Nebula lose energy by radiation and by expansion of the nebular volume. Because the electrons which radiate most of the energy have life times less than the age of the Nebula, about the same amount of relativistic particle energy as is lost has to be resupplied. It is a most striking result indeed that the loss of the rotational energy of the pulsar is of the same order as this energy requirement. Because no other comparable energy source appears to be available it seems that the rotational energy is transformed rather directly and highly efficiently into relativistic electron energy.

If we place the Nebula at a distance of $2 \mathrm{kpc}$ the radiative loss amounts to $1.6 \times$ $\times 10^{38} \mathrm{erg} / \mathrm{sec}$ (mainly in UV and very soft X-rays) and the expansion loss to $0.5 \times$ $\times 10^{38} \mathrm{erg} / \mathrm{sec}$ (for equipartition conditions) leading to a total electron energy requirement of $2 \times 10^{38} \mathrm{erg} / \mathrm{sec}$. The loss of rotational energy from the pulsar is about $5 \times 10^{38} \mathrm{erg} / \mathrm{sec}$ with an uncertainty of at least a factor of 3 due to uncertainties about the mass and detailed model of the neutron star. Comparing the energy requirement with the available energy we conclude that the efficiency for the acceleration of relativistic electrons cannot be much less than about 20 per cent. It follows from this that the ratio of the energy of protons and ions to that of electrons cannot be very large; the same qualitative conclusion is obtained from estimates of the total relativistic particle energy in the Nebula from the dynamics of the filamentary shell. This is in striking contrast with the cosmic-ray composition above $1 \mathrm{GeV}$ and makes it improbable that objects like the Crab are the main sources of cosmic rays.

The extremely high efficiency for electron acceleration imposes severe restrictions on the acceleration mechanism. Two such mechanisms have been recently proposed. Gunn and Ostriker (1969) have shown that the intense electromagnetic radiation, which would be present in a vacuum around the pulsar, should accelerate electrons to about $10^{13} \mathrm{eV}$, sufficient to explain the X-rays from the Crab. The energy attained by a unit charge is proportional to $\mathrm{m}^{1 / 3}$ and electrons would get about $8 \%$ of the energy of protons (or heavier particles). Alternatively in the model of Goldreich and Julian the electric fields around the pulsar would accelerate particles more or less electrostatically, probably resulting in a more equal distribution of the energy between electrons and positive particles. At the moment on the basis of these mechanisms we can perhaps understand the acceleration of the most energetic electrons. However the energy spectrum of the electrons - with most electrons having energies much smaller than the maximum - is not at all understood. But until a more self consistent model is forthcoming, it is difficult to say if this is a serious difficulty.

To summarize, it appears that the pulsar provides an adequate energy source for the Nebula and in a very qualitative way we may have the beginnings of a theory for the transformation of the rotational energy of the pulsar into the energy of relativistic particles and magnetic fields. 


\section{References}

Goldreich, P. and Julian, W. H.: 1969, Astrophys. J. 157, 869.

Gunn, J. E. and Ostriker, J. P.: 1969, Astrophys. J. 157, 1395.

\section{Discussion}

$J . P$. Ostriker: Our discussion of the acceleration of particles in the nebula concerned particles near the star. We have now looked at the effect of waves on the filaments and other material they may encounter. The accelerations are much weaker, but they have a different dependence on mass, namely an inverse square.

F. C. Michel: Some progress has been made towards self-consistent calculations of the magnetic field structure. Weber and Davis have made such calculations showing how the solar magnetic field extends into interplanetary space. These calculations can be made relativistic to apply to the pulsar problem, and one readily concludes that, if the surface fields are as large as generally proposed, then the nebula fields would be of the order of $10^{-3} \mathrm{G}$.

L. Mestel: A self-consistent model of the magnetic field is likely to differ in at least one important aspect from the solar field. In the Goldreich-Julian model the Alfvén speed is comparable with or greater than $c$, so that the regime is 'relativistic' even when the velocities of co-rotation near the star are well below $c$. It then follows that the field is not pulled out by the outflowing gas, but stays quasidipolar out to distances approaching the light-cylinder. This is in contrast to the solar case, where those fieldlines that link up the Sun with the interplanetary medium acquire a nearly radial structure soon after emerging from the solar surface.

N. Visvanathan: Observations of linear polarisation in the pulses of the pulsar (in optical wavelength) and the polarisation observation around pulsar show the direction of rotation axis of the pulsar and the direction of polarisation in the nebula agree well. The implication is strong that the magnetic field, at least in its vicinity may have been produced by the pulsar itself in some stage of its history. 